\title{
Radiative magneto-hydrodynamics in massive star formation and accretion disks
}

\author{
Rolf Kuiper, Mario Flock and Hubert Klahr \\ Max-Planck Institut für Astronomie, \\ Königstuhl 17, D-69117 Heidelberg, Germany \\ email: kuiper@mpia.de
}

\begin{abstract}
We briefly overview our newly developed radiation transport module for MHD simulations and two actual applications. The method combines the advantage of the speed of the Flux-Limited Diffusion approximation and the high accuracy obtained in ray-tracing methods.
\end{abstract}

Keywords. Radiative transfer - MHD - stars: formation - accretion disks - methods: numerical

\section{Radiative MHD}

Aim of the development of the radiation transport module described here is to achieve a fast method for approximative frequency-dependent radiation transport for (magneto-) hydrodynamical simulations in studies of the environment around a single object source.

The radiation transport module provides a Flux-Limited Diffusion (hereafter FLD) solver in cartesian, cylindrical and spherical coordinates with a potentially non-equidistant grid spacing in the first dimension, e.g. logarithmic in the radial coordinate. Additionally to the FLD approximation the module consists of a first order ray-tracing technique along the first dimension to account for single source irradiation (e.g. stellar heating of a proto-planetary disk or radiative feedback in massive star formation).

The local radiation field is split into two parts, originated from an externally irradiated (frequency-dependent) flux $\vec{F}(\nu)$ and a diffuse radiation energy density $E_{R}$, which are in equlibrium with the radiation from the dust grains:

$$
a T^{4}=E_{R}+\frac{1}{c \kappa_{P}(T)} \int \kappa(\nu)|\vec{F}(\nu)| d \nu,
$$

where $a$ is the radiation constant, $c$ is the speed of light, $\kappa(\nu)$ and $\kappa_{P}(T)$ represent the frequency-dependent and the Planck mean opacity for a given temperature $T$ respectively. Gas and dust temperatures are assumed to be the same. The irradiation is treated as an instantaneous source of additional energy, calculated via a first order ray-tracing as a function of the optical depth $\tau_{\nu}(r)$ and distance $r$ from the central star:

$$
\vec{F}(\nu, r)=\vec{F}_{*}(\nu)\left(\frac{R_{*}}{r}\right)^{2} e^{-\tau_{\nu}(r)}
$$

The evolution of the radiation energy is described by a Flux-Limited Diffusion equation

$$
\partial_{t} E_{R}=-f_{c}\left(\vec{\nabla} \cdot\left(D \vec{\nabla} E_{R}+\int \vec{F}(\nu, r) d \nu\right)-Q^{+}\right)
$$

with $f_{c}=\left(c_{v} \rho / 4 a T^{3}+1\right)^{-1}, D=\lambda c / \kappa_{R} \rho$ and $Q^{+}=-p \vec{\nabla} \cdot \vec{v}+$ additional source terms from hydrodynamics. The flux-limiter $\lambda$ is chosen according to Levermore \& Pomraning (1981), $c_{v}$ is the specic heat capacity, $\rho, p$ and $\vec{v}$ the gas density, thermal pressure and dynamical velocity and $\kappa_{R}$ specifies the Rosseland mean opacity. Scattering is neglected. 


\section{Application I: Massive Star Formation}

Due to the short Kelvin-Helmholtz contraction timescale of a massive star (Shu et al. 1987) the accretion process onto such a star is described by the interaction of the gravitationally forced inflow of matter with the radiative force escaping from the newly born star. The conservation of angular momentum leads to the formation of an accretion disk as well as polar cavities. At present we're studying this collapse scenario with respect to the effect of dimensionality (from 1 to 3D) and different applied physics (isothermal and adiabatic test runs, realistic cooling and frequency-averaged as well as frequencydependent radiative feedback).

\section{Application II: Accretion Disks}

We perform accretion disk simulations with a hydrodynamical stable stratified disk model $\left(\frac{H}{R}=0.05\right)$ and an initial toroidal magnetic field. The initial plasma beta $\beta$ is constant everywhere in the disk at a value of 25, the runs by Fromang \& Nelson (2006) (hereafter $\mathrm{FN}$ ). The convergence tests have a radial extension from 4 to $6 \mathrm{AU}, \pm 1$ scale height as vertical and $\pi / 3$ as azimuthal extension. We choose perodic boundaries in vertical and azimuthal direction. Small velocity perturbations in radial and vertical direction about $10^{-4} c_{0}$ initiate the non-linear MRI evolution. With the used high-order MHD Riemann solver (Mignone, et al. 2007) we get a converged alpha value of about $10^{-2}$ presented in figure 1 . In opposite, $\mathrm{FN}$ got an increasing alpha value from low $\alpha=10^{-3}$ to high $\alpha=5 * 10^{-3}$ (yet still not converged) with increasing resolution for a non-Riemann solver. In the future we will use the radiative module to calculate proper
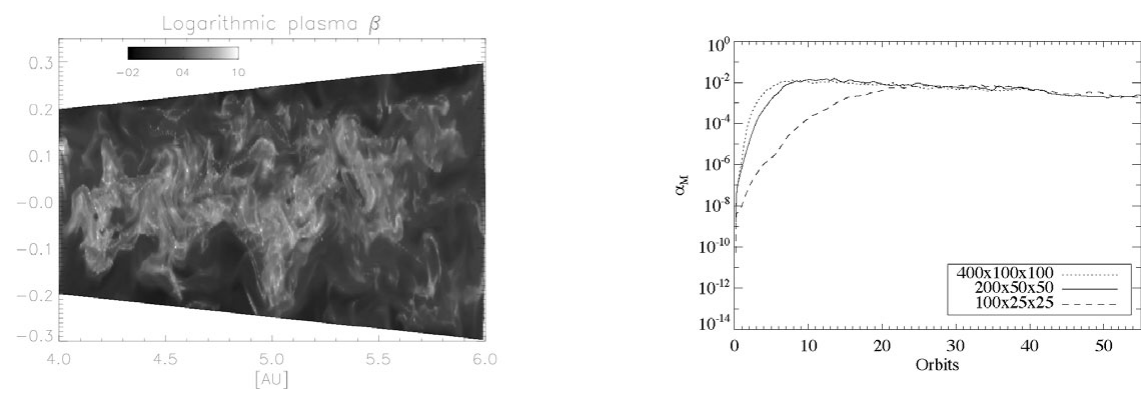

Figure 1. Left: Azimuthally averaged logarithmic plasma beta in the turbulent phase. Right: Evolution of Maxwell alpha at different resolution, converging against each other.

resistive terms $\eta(T, \rho)$ and to handle correctly the resulting heating and cooling processes of MHD turbulence and the behavior for the alpha stresses.

\section{References}

Levermore, C. D. \& Pomraning, G. C. 1981, ApJ 248, 321

Fromang, S. \& Nelson, A. P. 2006, A\& A 457, 343

Mignone, A., Bodo, G., Massaglia, S. Matsakos, T., Tesileanu, O., Zanni, C., \& Ferrari, A. 2007, ApJ 170,228

Pascucci, I., Wolf, S., Steinacker, J., et al. 2004, A\&A 417, 793

Shu, F. H., Adams, F. C., \& Lizano, S. 1987, ARAA 25, 23 